\title{
Epidermal Growth Factor Receptor Tyrosine Kinase Inhibitors As Adjuvant Therapy in Completely Resected Non-Small-Cell Lung Cancer
}

\author{
Silvia Novello, University of Turin, Torino, Italy
}

See accompanying article on page 4007

More than 10 years ago, the role of adjuvant chemotherapy in early stage non-small-cell lung cancer (NSCLC) was definitively established for stage II and III disease, ${ }^{1-6}$ whereas subset analyses suggested a benefit in patients with large IB tumors. ${ }^{7}$ Currently, any attempt to improve results of cisplatin-based chemotherapy by means of pharmacogenomics approaches has failed, and results of additional studies are eagerly awaited. ${ }^{8-10}$

In the article that accompanies this editorial, Kelly et $\mathrm{al}^{11}$ report the mature data of the RADIANT trial. It is noteworthy that only $51 \%$ of subjects in the erlotinib arm and 57\% in the placebo arm received adjuvant chemotherapy. This situation was quite likely related to the relevant proportion of enrolled patients with stage IB disease for which the value of adjuvant chemotherapy is still debatable in the absence of prospective data for large IB tumors.

No statistically significant difference was shown in disease-free survival (DFS), which was the primary endpoint of the study, whereas a trend suggestive of improved outcomes with erlotinib was demonstrated in the subgroup of patients (16.5\%) with an epidermal growth factor receptor (EGFR) -sensitizing mutation. EGFR mutation status was not among the stratification factors. In addition, an imbalance was observed in some patients' characteristics, such as performance status, in the comparison of the control arm in the EGFR mutant subgroup with the intention-to-treat population. Therefore, no definitive conclusion can be drawn.

The choice of DFS as the primary endpoint deserves comment. Although the correlation between progression-free survival (PFS) and overall survival (OS) has been demonstrated for advanced NSCLC, in the recognition of PFS as an acceptable surrogate endpoint for OS, ${ }^{12}$ the same information is not available for the early-disease setting. For adjuvant studies, OS remains the preferred primary endpoint, and DFS may be considered only if a futility-based interim analysis is planned to drive continuation of the trial or not.

The selection of patients was based on fluorescence in situ hybridization and EGFR immunohistochemistry, which are less sensitive tests for biomarkers than mutation testing. In the IPASS (Iressa Pan-Asia Study) trial, it was shown that the PFS benefit in patients with EGFR high copy number was entirely driven by the mutationpositive subgroup. In the era of personalized therapy, it is challenging to appropriately design a clinical trial that will preserve overtime the validity of the study hypothesis, especially in the adjuvant setting, where prolonged follow-up is needed. On another note, the recent revision of the lung cancer staging system with significant changes in stage allocation, and with further changes anticipated, represents an additional layer of complexity in the data interpretation.

Given the existing evidence, one reasonable clinical question could be, "Should all patients with resected adenocarcinoma be tested for EGFR mutation?” The College of American Pathologists/International Association for the Study of Lung Cancer/Association for Molecular Pathology expert consensus opinion, ${ }^{13}$ endorsed by the American Society of Clinical Oncology, ${ }^{14}$ encourages EGFR and anaplastic lymphoma kinase testing at diagnosis for all patients with early-stage adenocarcinoma. The benefit of this approach is that it enables rapid initiation of treatment in patients who experience a recurrence because molecular information is immediately available. This also favors their enrollment onto clinical trials, and a definitive trial to evaluate erlotinib and crizotinib in molecularly selected patients is planned in the United States (ALCHEMIST trial; NCT02201992, NCT02193282). Other randomized trials are ongoing in China (ADJUVANT; CTONG 1104) and Japan (IMPACT; WJOG6401L) in patients with completely resected IIIA-II/N2-N1 NSCLC with EGFR mutation who are randomly assigned to receive gefitinib versus a combination of vinorelbine plus platinum as adjuvant treatment. The disadvantage is the extra cost incurred by molecular testing of patients with early-stage disease who do not experience a relapse, as well as the possibility that the molecular profile could change at the time of relapse.

At the present time, the role of targeted agents as adjuvant therapies remains largely unknown. Patients with sensitizing mutations might derive more benefit from adjuvant chemotherapy, ${ }^{15}$ and chemotherapy may reduce the frequency of EGFR sensitizing mutations, suggesting a preferred response of EGFR mutated subclones to chemotherapy. ${ }^{16}$ In a Japanese phase III study, investigators administered adjuvant gefitinib or placebo for 2 years to patients with completely resected NSCLC in stage IB to IIIA for 4 to 6 weeks after surgery until recurrence or withdrawal. Recruitment was stopped after 38 patients were randomly assigned; this was because of interstitial lung disease. ${ }^{17}$ In another phase III study, ${ }^{18}$ researchers compared gefitinib with placebo after cisplatin-based chemotherapy. This study was prematurely closed as consequence of the negative 
outcomes of other phase III studies, ${ }^{19,20}$ but no advantage was detected in few enrolled patients with EGFR mutant tumors. ${ }^{21}$

The onset of acquired resistant mutations could be a concern because they have been frequently observed in EGFR-mutant, advanced NSCLC treated with EGFR tyrosine kinase inhibitors (TKIs). In a retrospective study, T790 resistant mutations were more common in cancers that recurred while patients were receiving an adjuvant EGFR TKI than in cancers that recurred after the EGFR TKI was stopped $(67 \% v 0 \%)$. Patients who had a recurrence after the EGFR TKI was stopped were treated again, with significant clinical benefit. In a large, prospective phase II study, patients with completely resected EGFR mutant tumors received erlotinib for 2 years after completion of adjuvant chemotherapy. In this trial, $63 \%$ of patients with recurrence underwent repeat biopsy; in only one case was a T790M resistance mutation detected, and the recurrent cases were still sensitive to EGFR TKIs.

The optimal duration of adjuvant therapy with EGFR TKIs remains a matter of debate. So far, in all adjuvant studies to test EGFR TKIs, the planned duration of therapy has been 2 years, but no statement can be made in this context. Despite the favorable toxicity profile of EGFR TKIs, no robust data are available for long-time exposure, and it is unknown how the duration of therapy might correlate with efficacy in this setting. Data from long-term survivors with advanced NSCLC treated with EGFR TKIs indicate a good quality of life and safety, even after $>3$ years of treatment. ${ }^{22}$ In the RADIANT study, the median duration of treatment was only 11.9 months for erlotinibtreated patients, and the rate of grade $\geq 3$ rash, diarrhea, and dosage reductions and interruptions were significantly higher in the experimental arm. Overall, the safety profile did not differ among EGFRmutant NSCLCs. These findings should be carefully considered in the plans for future studies involving patients who might potentially be cured with surgery plus adjuvant chemotherapy.

In summary, this study represents an additional piece of research that aims to define the best approach in patients with completely resected NSCLC and to define the role of EGFR TKIs in this setting. The authors should be praised for their foresight in designing this type of trial nearly 10 years ago. The efficacy of adjuvant EGFR TKIs in patients with mutations must be further investigated in well-designed randomized trials, such as the already-recruiting ALCHEMIST study or the randomized phase III clinical trials in EGFR mutation-positive patients initiated in China (ADJUVANT; CTONG 1104) and Japan (IMPACT; WJOG6401L).

\section{AUTHOR'S DISCLOSURES OF POTENTIAL CONFLICTS OF INTEREST}

Disclosures provided by the authors are available with this article at www.jco.org.

\section{REFERENCES}

1. Scagliotti GV, Fossati $R$, Torri $V$, et al: Randomized study of adjuvant chemotherapy for completely resected stage I, II or IIIA non-small cell lung cancer. J Natl Cancer Inst 95:1453-1461, 2003

2. Arriagada R, Bergman B, Dunant $A$, et al: Cisplatin-based adjuvant chemotherapy in patients with completely resected non-small cell lung cancer. N Engl J Med 350:351-360, 2004

3. Waller D, Peake MD, Stephens RJ, et al: Chemotherapy for patients with non-small cell lung cancer. The surgical setting of the Big Lung Trial. Eur $\mathrm{J}$ CardioThorac Surg 26:173-182, 2004

4. Douillard JY, Rosell R, Delena M, et al: Adjuvant vinorelbine plus cisplatin versus observation in patients with completely resected stage IB-IIIA non-small- cell lung cancer (Adjuvant Navelbine International Trialist Association [ANITA]): A randomised controlled trial. Lancet Oncol 7:719-727, 2006

5. Strauss GM, Herndon JE II, Maddaus MA, et al: Adjuvant paclitaxel plus carboplatin compared with observation in stage IB non-small-cell lung cancer: CALGB 9633 with the Cancer and Leukemia Group B, Radiation Therapy Oncology Group, and North Central Cancer Treatment Group Study Groups. J Clin Oncol 26:5043-5051, 2008

6. Winton T, Livingston R, Johnson $D$, et al: Vinorelbine plus cisplatin vs. observation in resected non-small-cell lung cancer. N Engl J Med 352:2589-2597, 2005

7. He J, Shen J, Yang C, et al: Adjuvant chemotherapy for the completely resected stage IB nonsmall cell lung cancer: A systematic review and metaanalysis. Medicine 94:e903, 2015

8. Wislez M, Barlesi $F$, Besse $B$, et al: Customized adjuvant phase II trial in patients with non-small-cell lung cancer: IFCT-0801 TASTE. J Clin Oncol 32: 1256-1261, 2014

9. Bepler G, Williams C, Schell MJ, et al: Randomized international phase III trial of ERCC1 and RRM1 expression-based chemotherapy versus gemcitabine/carboplatin in advanced non-small-cell lung cancer. J Clin Oncol 31:2404-2412, 2013

10. Massuti, $B$, Cobo M, Rodriguez-Paniagua $M$, et al: Randomized phase III trial of customized adjuvant chemotherapy (CT) according BRCA-1 expression levels in patients with node positive resected non-small cell lung cancer (NSCLS) SCAT: A Spanish Lung Cancer Group trial (Eudract:2007-000067-15; NCTgov: 00478699). J Clin Oncol 33:401s, 2015 (suppl; abstr 7507)

11. Kelly K, Altorki NK, Eberhardt WEE, et al: Adjuvant erlotinib versus placebo in patients with stage IB-IIIA non-small-cell lung cancer (RADIANT): A randomized, double-blind, phase III trial. J Clin Oncol 33:4007-4015, 2015

12. Shyr $Y$, Horn L, Berry L, et al: Are we making progress in lung cancer using progression-free survival as a surrogate endpoint? JAMA Oncol 1:202-203, 2015

13. Lindeman NI, Cagle PT, Beasley MB, et al: Molecular testing guideline for selection of lung cancer patients for EGFR and ALK tyrosine kinase inhibitors: Guideline from the College of American Pathologists, International Association for the Study of Lung Cancer, and Association for Molecular Pathology. Arch Pathol Lab Med 137:828-860, 2013

14. Leighl NB, Rekhtman N, Biermann WA, et al: Molecular testing for selection of patients with lung cancer for epidermal growth factor receptor and anaplastic lymphoma kinase tyrosine kinase inhibitors: American Society of Clinical Oncology endorsement of the College of American Pathologists/International Association for the Study of Lung Cancer/Association for Molecular Pathology guideline. J Clin Oncol 32:3673-3679, 2014

15. Tsao MS, Sakurada A, Ding K, et al: Prognostic and predictive value of epidermal growth factor receptor tyrosine kinase domain mutation status and gene copy number for adjuvant chemotherapy in non-small cell lung cancer. $J$ Thor Oncol 6:139-147, 2011

16. Bai $\mathrm{H}$, Wang Z, Chen $\mathrm{K}$, et al: Influence of chemotherapy on EGFR mutation status among patients with non-small-cell lung cancer. J Clin Oncol 30:30773083, 2012

17. Tsuboi M., Kato H, Nagai K, et al: Gefitinib in the adjuvant setting: Safety results from a phase III study in patients with completely resected non-small cell lung cancer. Anticancer Drugs 16:1123-1128, 2005

18. Goss GD, O'Callaghan C, Lorimer I, et al: Gefitinib versus placebo in completely resected non-small-cell lung cancer: Results of the NCIC CTG BR19 study. J Clin Oncol 31:3320-3326, 2013

19. Kelly K, Chansky K, Gaspar LE, et al: Phase III trial of maintenance gefitinib or placebo after concurrent chemoradiotherapy and docetaxel consolidation in inoperable stage III non-small-cell lung cancer: SWOG S0023. J Clin Oncol 26:2450-2456, 2008

20. Thatcher N, Chang A, Parikh P, et al: Gefitinib plus best supportive care in previously treated patients with refractory advanced non-small-cell lung cancer: Results from a randomised, placebo-controlled, multicentre study (Iressa Survival Evaluation in Lung Cancer). Lancet 366:1527-1537, 2005

21. Goss GD, O'Callaghan C, Lorimer I, et al: Gefitinib versus placebo in completely resected non-small-cell lung cancer: Results of the NCIC CTG BR19 study. J Clin Oncol 31:3320-3326, 2013

22. Li L, Zhong W, Liao M, et al: A study on the long-term non-small cell lung cancer survivors in the Expand Access Program of gefitinib in China. Zhongguo Fei Ai Za Zhi 15:332-339, 2012

DOI: 10.1200/JCO.2015.63.7587; published online ahead of print at www.jco.org on October 5, 2015 


\section{AUTHOR'S DISCLOSURES OF POTENTIAL CONFLICTS OF INTEREST}

Epidermal Growth Factor Receptor Tyrosine Kinase Inhibitors As Adjuvant Therapy in Completely Resected Non-Small-Cell Lung Cancer

The following represents disclosure information provided by authors of this manuscript. All relationships are considered compensated. Relationships are self-held unless noted. I = Immediate Family Member, Inst = My Institution. Relationships may not relate to the subject matter of this manuscript. For more information about ASCO's conflict of interest policy, please refer to www.asco.org/rwc or jco.ascopubs.org/site/ifc.

Silvia Novello

Speakers' Bureau: MSD Oncology, Eli Lilly, Boehringer Ingelheim,

Roche, AstraZeneca 